\title{
Parameter Estimation of Photovoltaic Models via Cuckoo Search
}

\author{
Jieming Ma, ${ }^{1,2}$ T. O. Ting, ${ }^{2}$ Ka Lok Man, ${ }^{2}$ Nan Zhang, \\ Sheng-Uei Guan, ${ }^{2}$ and Prudence W. H. Wong ${ }^{1}$ \\ ${ }^{1}$ Department of Computer Science, University of Liverpool, Ashton Building, Ashton Street, Liverpool, L69 3BX, UK \\ ${ }^{2}$ Xian Jiaotong-Liverpool University, 111 Ren'ai Road, Suzhou Dushu Lake, HET, Jiangsu Province 215123, China
}

Correspondence should be addressed to T. O. Ting; toting@xjtlu.edu.cn

Received 27 June 2013; Accepted 11 July 2013

Academic Editor: Xin-She Yang

Copyright (c) 2013 Jieming Ma et al. This is an open access article distributed under the Creative Commons Attribution License, which permits unrestricted use, distribution, and reproduction in any medium, provided the original work is properly cited.

Since conventional methods are incapable of estimating the parameters of Photovoltaic (PV) models with high accuracy, bioinspired algorithms have attracted significant attention in the last decade. Cuckoo Search (CS) is invented based on the inspiration of brood parasitic behavior of some cuckoo species in combination with the Lévy flight behavior. In this paper, a CS-based parameter estimation method is proposed to extract the parameters of single-diode models for commercial PV generators. Simulation results and experimental data show that the CS algorithm is capable of obtaining all the parameters with extremely high accuracy, depicted by a low Root-Mean-Squared-Error (RMSE) value. The proposed method outperforms other algorithms applied in this study.

\section{Introduction}

Photovoltaic (PV) cells, normally assembled into modules or arrays on mounting systems, are capable of producing electrons when photons strike its surface. Taking the advantages of many promising features like renewability, less pollution, and ease of installation, PV generators are envisaged to be an important energy source for the future.

Due to the high initial cost of a PV-supplied system, predictive performance tools are widely used by engineers to optimize the system performance $[1,2]$. PV manufacturers normally provide limited tabular data measured under the Standard Test Conditions (STCs), which correspond to a cell temperature of $25^{\circ} \mathrm{C}$ and an irradiance of $1000 \mathrm{~W} / \mathrm{m}^{2}$ at 1.5 air mass spectral distributions. As reported in [3], PV generators always operate under environments far from the STCs. Due to this reason, the data available in the datasheet usually fail to fulfill the engineering requirements.

PV model, with the ability to predict $I-V$ characteristics of PV generators under an operating environment other than the STCs, is a predictive performance tool that allows consumers to maximize the cost effectiveness of the system before installation [2]. They are generally analytical equations based on a physical description that formulate PV generated current $(I)$ with the most crucial technical characteristics and the environmental variables, such as the operating voltage $(V)$, the ambient temperature $(T)$, and the irradiance $(G)$. Over the years, significant research efforts have been contributing to the development of the behavioral models [48]. Among numerous modeling approaches, the Single-Diode Model (SDM) is the most widely utilized PV model in the literature. A general SDM includes five parameters, namely, photocurrent $\left(I_{\mathrm{pv}}\right)$, saturation current $\left(I_{o}\right)$, diode ideality constant $(n)$, series resistance $\left(R_{s}\right)$, and shunt resistance $\left(R_{p}\right)$. In order to adapt PV model behavior to different operating conditions, de Blas et al. [9] suggested to apply the procedure described in the International Standard IEC 891 that relates current and voltage of the PV characteristics at given values of $T$ and $G$ with the corresponding values at different operating environments. The Improved Single Diode Model (ISDM) presented by De Soto et al. [5] includes the dependence of the PV parameters on operating conditions. The normal parameters at the STCs are necessary to be determined in this model. Both SDM and ISDM are adopted in this study of parameter estimation.

Analytical methods [5, 10-12] are common approaches in estimating the parameters by mathematical equations. Although having the merit of simplicity, it is hard to further reduce the errors of the estimated values. Furthermore, analytical methods utilize the $I-V$ curve features or 
semiconductor parameters that are unavailable in the datasheet. This problem often reduces its feasibility. Recently, $\mathrm{PV}$ parameter estimation is deemed as a multidimensional optimization problem. Several computational intelligence methods, such as Genetic Algorithms (GA) [13], Chaos Particle Swarm Optimization (CPSO) [14], Firefly [15], and Pattern Search (PS) [16], were proposed in the literature. These algorithms usually extract relevant parameters by minimizing the Root Mean Square Error (RMSE) as the objective function in the optimization process. Askarzadeh and Rezazadeh [17] reported that the optimization methods produce better results than analytical methods.

Cuckoo Search (CS) is a nature-inspired optimization algorithm based on the fascinating breeding behavior such as brood parasitism of certain species of cuckoos. In [18, 19], Yang and Deb reported that the CS algorithm outperforms Particle Swarm Optimization (PSO) and GA algorithms for various standard test functions. In this paper, a CS-based parameter estimation method for the SDM and ISDM is presented. Simulation and experimental results show superior accuracy and feasibility of the proposed parameter estimation method.

The rest of the paper is organized as follows. Section 2 explains both PV models (SDM and ISDM) used in this work. The objective function formulation is given in Section 3. This is followed by results and discussions in Section 4. The results comparison is also available here, and finally the conclusions are derived in Section 5.

\section{PV Modeling}

2.1. Single Diode PV Model (SDM). PV cells are made of a variety of semiconductor materials using different manufacturing processes. The working principle of PV cells is essentially on the basis of the PV effect, which refers to the generation of a potential difference at the $P-N$ junction in response to visible or other radiation. When a PV cell is exposed to light, the semiconductor materials absorb photons, and accordingly charged carriers are generated. Potential difference and current in the external circuit lead to the separation of carriers in the internal electric field created by the $P-N$ junction and collection at the electrodes. The photogenerated charge carriers can be subsequently captured in the form of an electric current, that is, electricity $I_{\mathrm{pv}}$. Eliminated the PV effect, a PV cell behaves like a conventional diode that does not depend on any light parameters. The Shockley diode equation is generally used to describe the current flowing through the diode $\left(I_{d}\right)$ :

$$
I_{d}=I_{o}\left(e^{V_{d} / n V_{t}}-1\right) .
$$

In (1), $I_{o}$ is the normal diode current, and $V_{d}$ represents the electrical potential difference between the two ends of the diode. The ideality factor $n$ is assumed to be independent of the environment variables $T$ and $G$. $V_{t}$ denotes the thermal voltage of the $\mathrm{PV}$, and its value can be written as a function of $T$ :

$$
V_{t}=\frac{k T}{q}
$$

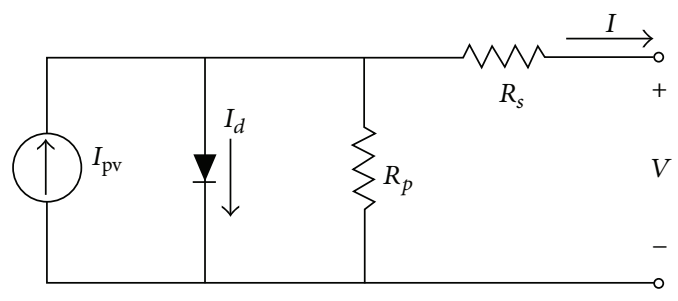

FIGURE 1: The equivalent circuit of the SDM.

where $k$ and $q$ represent the Boltzmann constant $(1.380650$ $\left.\times 10^{-23} \mathrm{~J} / \mathrm{K}\right)$ and the electron charge $\left(1.602176 \times 10^{-19} \mathrm{C}\right)$, respectively.

SDM assumes that the superposition principle holds; that is, the total characteristic is the sum of the dark and illuminated characteristics [3-5]. As expressed in (3) below, the terminal current $I$ is therefore equal to $I_{\mathrm{pv}}$ subtracting the current diverting through the diode and $R_{s}$. The equivalent circuit of the SDM is shown in Figure 1.

$$
I=I_{\mathrm{pv}}-I_{o}\left(e^{\left(V+I R_{s}\right) / n V_{t}}-1\right)-\frac{V+I R_{s}}{R_{p}} .
$$

PV module is a particular case of the PV cells connected in series. If the number of the connected cells is up to $N_{s}, V_{t}$ is scaled to $N_{s}$ times. The model equation is then rewritten as

$$
I=I_{\mathrm{pv}}-I_{o}\left(e^{\left(V+I R_{s}\right) / n N_{s} V_{t}}-1\right)-\frac{V+I R_{s}}{R_{p}} .
$$

In this sense, $I_{\mathrm{pv}}, I_{o}, R_{s}$, and $R_{p}$ are the corresponding parameters of a PV module.

2.2. Improved Single Diode Model (ISDM). The traditional SDM ignores the operating conditions effect on these parameters. However, some studies have shown that the parameters, such as $I_{\mathrm{pv}}$ and $I_{o}$, vary under different environmental conditions. These are due to changes of temperature $T$ and irradiance $G$. Aiming to evaluate the PV behavior at environmental conditions other than the normal values $T_{n}$ and $G_{n}$, the relations between the operating parameters and the normal parameters are studied by numerous researchers.

In [4], the value of light-generated $I_{\mathrm{pv}}$ is reported to be linearly dependent on the solar irradiation under the influence of temperature:

$$
I_{\mathrm{pv}}=\frac{G}{G_{n}}\left(I_{\mathrm{pv} n}-K_{i} \Delta T\right),
$$

where $I_{\mathrm{pv} n}$ is the light-generated current at the STCs. $K_{i}$, the short-circuit current coefficient, is one of the ISDM parameters. The difference between the standard test temperature $T_{n}$ and $T$ is denoted by $\Delta T$.

Based on the diode theory, Messenger and Ventre [20] presented an approximate linear expression for the diode saturation current $I_{o}$, which can be expressed as

$$
I_{o}=I_{o n}\left(\frac{T}{T_{n}}\right) e^{\left[\left(q E_{g} / n k\right)\left(1 / T_{n}-1 / T\right)\right]},
$$


where $E_{g}$ is the material band gap. Usually, $E_{g}$ is set at a reasonable level depending on the semiconductor materials $(1.12 \mathrm{eV}$ for crystalline silicon and $1.75 \mathrm{eV}$ for amorphous silicon) in simulation and design tools [21]. De Soto et al. [5] present an estimation method for $E_{g}$ in a wide temperature range:

$$
E_{g}=E_{g n}(1-0.0002677 \Delta T),
$$

where $E_{g n}$ is a normal value at the STCs $\left(E_{g n}=1.12 \mathrm{eV}\right.$ for silicon cells and $E_{g n}=1.6 \mathrm{eV}$ for the triple junction amorphous cells).

In [3], Lo Brano et al. study how the series and shunt resistances are affected by the solar irradiance. On the basis of the experimental data, the values of $R_{s}$ and $R_{p}$ are observed varying in inverse linear modes with $G$ :

$$
\begin{aligned}
& R_{s}=\frac{G_{n}}{G} R_{s n}, \\
& R_{p}=\frac{G_{n}}{G} R_{p n},
\end{aligned}
$$

where the values of the resistances $R_{s n}$ and $R_{p n}$ are evaluated under the STCs.

By using the aforementioned relations, the ISDM described in [5] is able to analytically describe the $I-V$ characteristic of a PV generator for each generic condition of operative temperature and solar irradiance.

\section{Parameter Estimation}

3.1. Formulation of Parameter Estimation Problem. PV parameter estimation is a process that minimizes the difference between the measured data and the calculated current by adjusting the normal PV parameters. When the number of experimental data is up to $N$, the objective function can be formulated by RMSE as

$$
\text { RMSE }=\sqrt{\frac{1}{N} \sum_{i=1}^{N}\left(f_{i}(V, I, x)\right)^{2}},
$$

where $x=\left[I_{\mathrm{pv}}, I_{o} n, R_{s}, R_{p}\right]$ for $\mathrm{SDM}$ and $x=$ $\left[I_{\mathrm{pv} n}, I_{o n}, n, R_{s n}, R_{p n}, K_{i}, E_{g}\right]$ for ISDM. $f(V, I, x)$ is the homogeneous form of (4) which expresses the $I-V$ characteristics of the SDM:

$$
f(V, I, x)=I_{\mathrm{pv}}-I_{o}\left(e^{\left(V+I R_{s}\right) / n N_{s} V_{t}}-1\right)-\frac{V+I R_{s}}{R_{p}}-I .
$$

For the case of ISDM, $I_{\mathrm{pv}}, I_{o}, n, R$, and $R_{p}$ satisfy the relational expressions discussed in the previous subsection, namely, (5)-(8).

3.2. Cuckoo Search. The CS algorithm [18, 19], proposed by Yang and Deb, is a nature-inspired stochastic global search algorithm that follows three idealized behavior rules.

(i) A cuckoo lays an egg and dumps it randomly into other bird species' nests.

\section{Cuckoo Search via Lévy Flights}

Initialization of $n$ host nests (population) while within the stopping criterion, Choose a cuckoo egg by Lévy flights and evaluate its fitness $\left(F_{i}\right)$;

Choose an egg in other's nest randomly and calculate its fitness $\left(F_{j}\right)$;

If $F_{i}>F_{j}$, replace $j$ th egg by $i$ th egg;

A fraction $\left(p_{a}\right)$ of worse nests are demolished and replaced by new ones; end while

Preserve good nests (best solutions).

Pseudocode 1: Pseudocode of the Cuckoo Search (CS) [19].

(ii) The best nests with high quality eggs will be carried forward to the next generation.

(iii) There are a fixed number of available host nests. If a host bird discovers that the eggs are not its own, it will either throw these alien eggs away, or it may abandon the nest and build a brand new nest at a nearby location.

Based on the three rules, the basic steps of CS can be summarized by the pseudocode shown in Pseudocode 1 . In the CS algorithm, a pattern corresponds to a nest while each individual attribute of the pattern corresponds to an egg laid by the cuckoo. On the basis of random-walk algorithms, the general system equation of the CS algorithm is given in:

$$
X_{g+1 ; i}=X_{g ; i}+\alpha \otimes \operatorname{Lévy}(\beta),
$$

where $g$ and $i$ denote the generation number $(g=$ $1,2,3, \ldots$, MaxGen) and the pattern number $(i=1,2, \ldots, n)$, respectively. The product $\otimes$ means entry-wise multiplications. Here $\alpha>0$ is the step size scaling factor which should be related to the scales of the problem of interest [19]. The $j$ th attributes of the $i$ th pattern is initiated by using (12),

$$
X_{g=0 ; j, i}=\operatorname{rand} \cdot\left(U b_{i}-L b_{i}\right)+L b_{i},
$$

where $U b_{i}$ and $L b_{i}$ are the upper and lower bounds of the $j$ th attributes, respectively. In each computation step, the CS algorithm checks whether the value of an attribute exceeds the allowed search range. If this happens, the value of the related attribute will be updated with the corresponding boundary value.

Before the searching process, the CS algorithm detects the most successful pattern as $x_{\text {best }}$ pattern. Among the existing algorithms exist for generating Lévy flights in the literature, Yang and Deb $[18,19]$ reported that Mantegna's algorithm [22] works well in most of the optimization problems. Accordingly the evolution phase of the pattern initialized with the detection step of $\phi$, which is given by (13) [23]:

$$
\phi=\left(\frac{\Gamma(1+\beta) \cdot \sin (\pi \cdot \beta / 2)}{\Gamma\left(((1+\beta) / 2) \cdot \beta \cdot 2^{(\beta-1) / 2}\right)}\right)^{1 / \beta},
$$


where $\beta$ is 1.5 in the standard software implementation of the CS algorithm [30]. $\Gamma$ denotes the gamma function.

After initialization, the evolution phase of the $x_{i}$ pattern starts by defining the donor vector $v$, where $v=x_{i}$. The required step size of the $j$ th attributes can be calculated by the following equation:

$$
s_{j}=0.01 \cdot\left(\frac{u_{j}}{v_{j}}\right)^{1 / \beta} \cdot\left(v-x_{\text {best }}\right),
$$

where $u=\phi \cdot \operatorname{rand} n[D]$ and $v=\operatorname{rand} n[D]$. The $\operatorname{rand} n[D]$ function generates a uniform integer between $[1, D][25]$. The donor pattern $v$ is then randomly adjusted by

$$
v=v+s_{j} \cdot \operatorname{rand} n[D]
$$

The CS algorithm will evaluate the fitness of the random pattern. If a better solution is caught, the $x_{\text {best }}$ pattern will be updated. The unfeasible patterns are revised by the crossover operator given in (16) as follows:

$$
v_{i}= \begin{cases}x_{i}+\operatorname{rand} \cdot\left(x_{r 1}-x_{r 2}\right), & \text { rand }_{i}>p_{0} \\ x_{i}, & \text { others }\end{cases}
$$

where $p_{0}$ is the mutation probability value $\left(p_{0}=0.25\right.$ in the standard software implementation [30]). The final step of a generation is to check if the revised infeasible patterns deliver a better solution.

\section{Results and Discussions}

With the aim of providing a thorough evaluation of the CS algorithm in estimating the PV parameters, both SDM and ISDM are considered in this paper. Two case studies are designed to estimate the CS algorithm in model parameters estimation:

(i) a commercial $57 \mathrm{~mm}$ diameter solar cell (R.T.C. France [26]) operating at the standard irradiance level;

(ii) a PV module (KC200GT Multicrystal Photovoltaic Module) operating under varied environment conditions.

During the parameter extraction process, the objective function $f(V, I, x)$ is minimized with respect to the parameters range. In theory, the value of $I_{\mathrm{pv} n}$ is slightly larger than that of $I_{\mathrm{scn}} . E_{g n}$ is in a loose range from $1 \mathrm{eV}$ to $2 \mathrm{eV} . K_{i}$ is around the value provided by the datasheet (normally less than $0.02 \% /{ }^{\circ} \mathrm{C}$ ). The $I_{o n}$ is usually less than $50 \mu \mathrm{A}$. As stated in [27], the ideality factor ranges between 1 and 2 . PV modules produced by most manufacturers have $R_{s}$ less than $0.5 \Omega$ and $R_{p}$ between 5 and $170 \Omega[8,28]$. As for PV cell, the ranges of $R_{s}$ and $R_{p}$ can be scaled by simply dividing $N_{s}[29]$.

Statistical analysis is performed to evaluate the quality of the fitted models to the experimental data. Besides RMSE, other two fundamental measures, namely, Individual Absolute Error (IAE) and the Mean Absolute Error (MAE), are
TABLE 1: A comparison between the parameter results obtained by the CS algorithm and that of other algorithms from the SDM.

\begin{tabular}{lcccc}
\hline & CS & CPSO [14] & GA [13] & PS [16] \\
\hline$I_{\mathrm{pv}}$ & 0.7608 & 0.7607 & 0.7619 & 0.7617 \\
$I_{o}$ & $3.23 E-07$ & $4.00 E-07$ & $8.09 E-07$ & $9.98 E-07$ \\
$n$ & 1.4812 & 1.5033 & 1.5751 & 1.6 \\
$R_{s}$ & 0.0364 & 0.0354 & 0.0299 & 0.0313 \\
$R_{p}$ & 53.7185 & 59.012 & 42.3729 & 61.1026 \\
RMSE & 0.0010 & 0.0014 & 0.0191 & 0.0149 \\
\hline
\end{tabular}

applied to evaluate in this paper. Equations (17) and (18) preset the IAE and MAE, respectively:

$$
\begin{gathered}
\mathrm{IAE}=\left|I_{\text {calculated }}-I_{\text {measured }}\right|, \\
\mathrm{MAE}=\frac{1}{N} \sum_{i=1}^{N} \mathrm{IAE}_{i} .
\end{gathered}
$$

The optimization algorithms applied in this paper are programmed in MATLAB. Similar simulation conditions, including population size, maximum generation number, and search ranges, are set to ensure a fair evaluation (population size $=25$; maximum generation number $=5000)$.

4.1. Case Study 1: Parameter Estimation for a PV Cell at the Certain Irradiance Level. Table 1 lists the model parameters of the R.T.C France PV cell at $33^{\circ} \mathrm{C}$, which are extracted from the experimental data in [26]. The parameters obtained from the CS algorithm are compared with three different parameter estimation approaches: CPSO [14], GA [13], and PS [16]. From the RMSEs of these methods, which are listed in the last row of Table 1, the CS algorithm [30] outperforms the other three optimization methods. CS obtained slightly lower RMSE, recording 0.0010 in numerical value.

During the parameter estimation process for the SDM, the values of the objective function in different optimization algorithms are shown in Figure 2. The function "ga" in MATLAB [31], whose crossover rate $P_{c}=0.8$ and mutation rate $P_{m}=0.2$, is utilized for the convergence process test. As for PSO implementation [24], the algorithm parameters are set as learning factors $c_{1}=c_{2}=2$, inertia factors $w_{\max }=0.9$, $w_{\min }=0.4$, and velocity clamping factor $V_{\max }=0.5$. In Figure 2, no further improvement by GA is observed after 500 iterations. On the contrary, the CS algorithm showed continuous improvement until the maximum generation. The CS algorithm, whose convergence speed is slightly faster than PSO, shows the best accuracy result in the minimization task after 5000 iterations.

Table 2 lists the parameters of the ISDM obtained by the CS algorithm. In order to evaluate the accuracy of the CSbased estimation, these parameters are substituted into the ISDM. Since the $I-V$ demonstrates nonlinear characteristics, the PV terminal current $I$ is solved by the Newton-Raphson method [32] in this paper. In Table 3, the calculated results $I_{\text {ISDM }}$ are compared with the experimental data $I_{\text {measured }}$ to observe the agreement between them. The notations IAE $_{\mathrm{SDM}}$ and IAE $_{\text {ISDM }}$ denote the IAE for SDM and ISDM, respectively. 


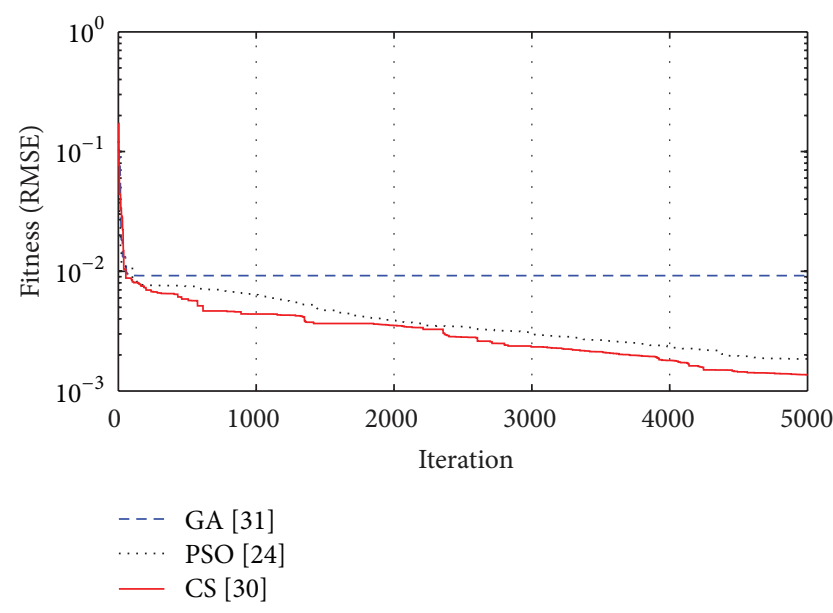

FIGURE 2: Convergence process of different optimization algorithms during the parameter estimation process of the SDM.

TABLE 2: Parameters of the ISDM obtained by the CS algorithm.

\begin{tabular}{lcccccc}
\hline$I_{\mathrm{pv} n}$ & $I_{\text {on }}$ & $n$ & $R_{\text {sn }}$ & $R_{\text {pn }}$ & $K_{i}$ & $E_{\text {gn }}$ \\
\hline 0.7361 & $1.84 E-07$ & 1.5009 & 0.0355 & 57.8394 & 0.0031 & 1.0020 \\
\hline
\end{tabular}

Although the RMSE of the ISDM is less than that of CPSO, GA, and PS, it is similar to the RMSE of the conventional SDM under a certain environmental condition.

4.2. Case Study 2: Parameter Estimation for a PV Module under Different Environment Conditions. In this section, the validity of the CS algorithm is evaluated using KC200GT PV module operating under different environment conditions. The estimated parameters, both in the SDM and ISDM, are shown in Table 4. As illustrated in Section 1, the main application of the parameter extraction is to predict the $I-V$ characteristics for design purpose. It is worth pointing out that the SDM parameters can only be extracted by the experimental data measured under a certain test condition. Significant errors may occur as the experimental data are measured under varying operating conditions. In the commercial simulation tool like PSIM [21], the PV parameters of the SDM are firstly estimated at the STCs, then the equations (given in the appendix) are applied to calculate the electrical characteristics of different operating conditions. The ISDMbased parameter estimation, however, can be performed by the data measured under any conditions.

Figure 3 shows the $I$ - $V$ curves generated using the parameters obtained by the CS algorithm. The simulated results are compared with the experimental data, which are collected at five different irradiance levels $\left(1000 \mathrm{~W} / \mathrm{m}^{2}, 800 \mathrm{~W} / \mathrm{m}^{2}\right.$, $600 \mathrm{~W} / \mathrm{m}^{2}, 400 \mathrm{~W} / \mathrm{m}^{2}$, and $200 \mathrm{~W} / \mathrm{m}^{2}$ ) and three different temperature levels $\left(25^{\circ} \mathrm{C}, 50^{\circ} \mathrm{C}\right.$, and $\left.75^{\circ} \mathrm{C}\right)$. It can be seen that the $I-V$ curves of the ISDM fit the whole range of the experimental dataset. On the other hand, the errors of SDM seem larger at lower irradiance and higher temperature levels. With the experimental data, the RMSE of the current $I$ in
TABLE 3: A comparison between the errors of ISDM and SDM. The parameters are extracted by the CS algorithm.

\begin{tabular}{lccccc}
\hline No & $V_{\text {measured }}$ & $I_{\text {measured }}$ & $I_{\text {ISDM }}$ & IAE $_{\text {ISDM }}$ & IAE $_{\text {SDM }}$ \\
\hline 1 & -0.2057 & 0.7640 & 0.7639 & 0.0001 & 0.0001 \\
2 & -0.1291 & 0.7620 & 0.7626 & 0.0006 & 0.0007 \\
3 & -0.0588 & 0.7605 & 0.7614 & 0.0009 & 0.0009 \\
4 & 0.0057 & 0.7605 & 0.7602 & 0.0003 & 0.0003 \\
5 & 0.0646 & 0.7600 & 0.7592 & 0.0008 & 0.0009 \\
6 & 0.1185 & 0.7590 & 0.7583 & 0.0007 & 0.0010 \\
7 & 0.1678 & 0.7570 & 0.7574 & 0.0004 & 0.0001 \\
8 & 0.2132 & 0.7570 & 0.7565 & 0.0005 & 0.0009 \\
9 & 0.2545 & 0.7555 & 0.7555 & 0.0000 & 0.0004 \\
10 & 0.2924 & 0.7540 & 0.7540 & 0.0000 & 0.0003 \\
11 & 0.3269 & 0.7505 & 0.7517 & 0.0012 & 0.0009 \\
12 & 0.3585 & 0.7465 & 0.7476 & 0.0011 & 0.0009 \\
13 & 0.3873 & 0.7385 & 0.7402 & 0.0017 & 0.0016 \\
14 & 0.4137 & 0.7280 & 0.7273 & 0.0007 & 0.0006 \\
15 & 0.4373 & 0.7065 & 0.7066 & 0.0001 & 0.0005 \\
16 & 0.4590 & 0.6755 & 0.6748 & 0.0007 & 0.0002 \\
17 & 0.4784 & 0.6320 & 0.6304 & 0.0016 & 0.0011 \\
18 & 0.4960 & 0.5730 & 0.5717 & 0.0013 & 0.0009 \\
19 & 0.5119 & 0.4990 & 0.4994 & 0.0004 & 0.0005 \\
20 & 0.5265 & 0.4130 & 0.4137 & 0.0007 & 0.0005 \\
21 & 0.5398 & 0.3165 & 0.3176 & 0.0011 & 0.0007 \\
22 & 0.5521 & 0.2120 & 0.2127 & 0.0007 & 0.0001 \\
23 & 0.5633 & 0.1035 & 0.1033 & 0.0002 & 0.0008 \\
24 & 0.5736 & -0.0100 & -0.0089 & 0.0011 & 0.0008 \\
25 & 0.5833 & -0.1230 & -0.1244 & 0.0014 & 0.0014 \\
26 & 0.5900 & -0.2100 & -0.2095 & 0.0005 & 0.0009 \\
\hline $\mathrm{MAE}$ & & & & 0.0007 & 0.0007 \\
$\mathrm{RMSE}$ & & & & 0.0010 & 0.0010 \\
\hline & & & & &
\end{tabular}

TABle 4: Parameters of the KC200GT PV module obtained by the CS algorithm.

(a) SDM parameters (extracted by the CS algorithm)

\begin{tabular}{lcccc}
\hline$I_{\mathrm{pv}}$ & $I_{\mathrm{o}}$ & $n$ & $R_{\mathrm{s}}$ & $R_{p}$ \\
\hline 8.1729 & $4.23 E-10$ & 1.0090 & 0.2665 & 140.4875 \\
\hline
\end{tabular}

(b) ISDM parameters (extracted by the CS algorithm)

\begin{tabular}{lcccccc}
\hline$I_{\mathrm{pv} n}$ & $I_{\text {on }}$ & $n$ & $R_{s n}$ & $R_{p n}$ & $K_{i}$ & $E_{g n}$ \\
\hline 8.1847 & $5.12 E-10$ & 1.0170 & 0.2574 & 117.9224 & 0.0028 & 1.2474 \\
\hline
\end{tabular}

SDM is calculated as 0.2837 , while the RMSE of $I$ in ISDM is only 0.0776 .

Figure 4 shows the absolute current errors of different performance predicting methods under different operating conditions. The curves denoted by the label "analytical SDM" are obtained from the analytical SDM model [4]. Ignoring the effect of incidence angle and air mass, the curves labeled by "analytical ISDM" denote the $I-V$ curves from De Soto's 


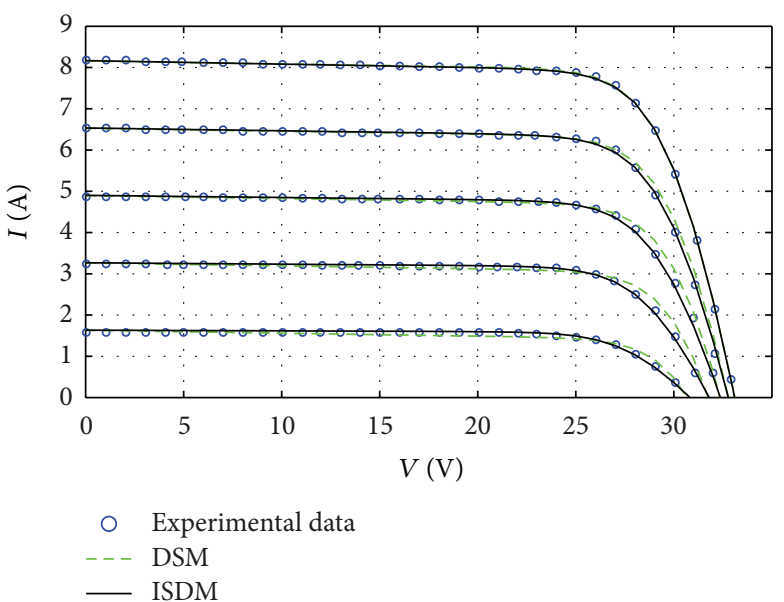

(a)

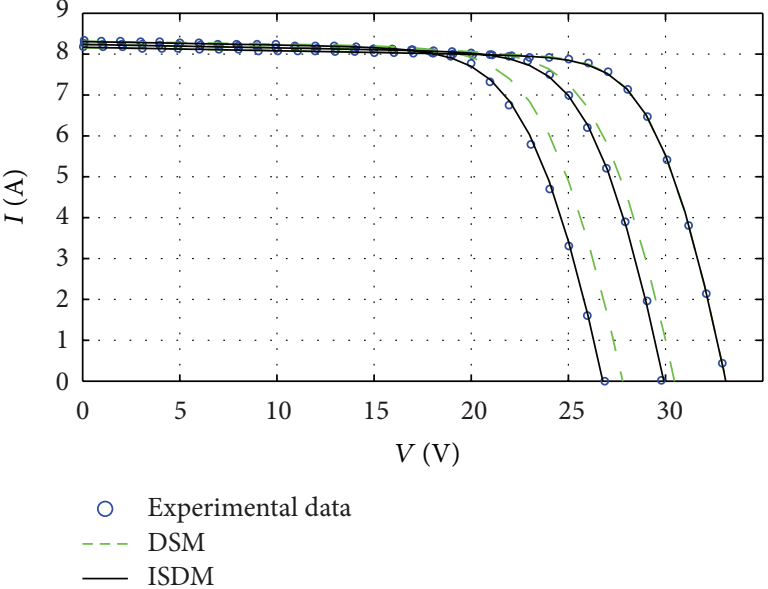

(b)

FIgURE 3: The simulated $I-V$ characteristic curves of the KC200GT PV module: (a) under different irradiance levels; (b) under different temperature levels.

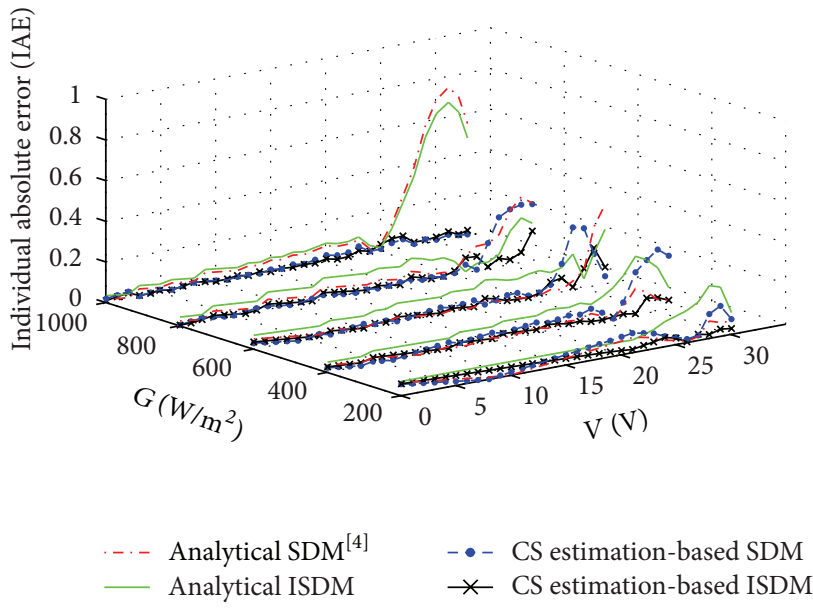

(a)

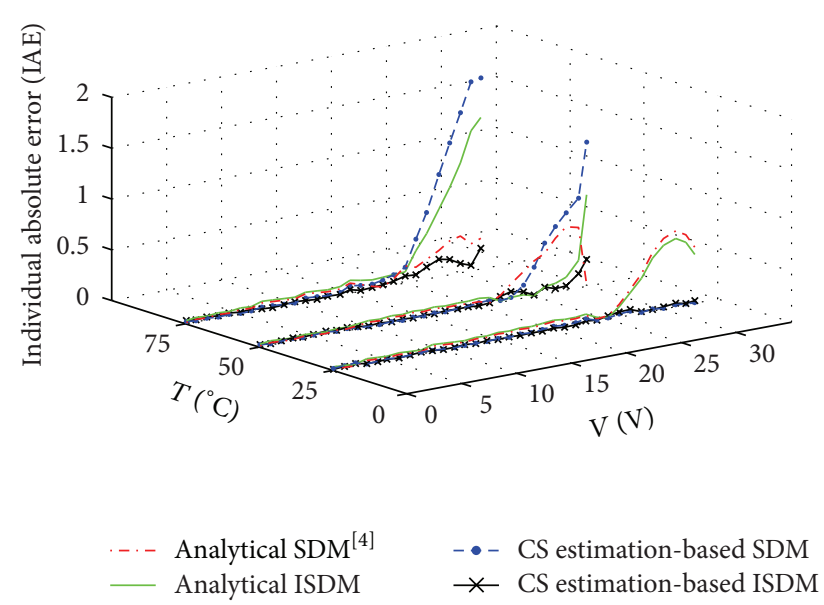

(b)

FIGURE 4: A comparison of the individual absolute errors among different PV modeling methods: (a) under different irradiance levels; (b) under different temperature levels.

analytical ISDM model [5]. It is evident the ISDM with the parameters extracted by the CS algorithm is more accurate than the analytical model. As for the SDM, the CS algorithm is capable of extracting a set of PV parameters with a good fit for the experimental data at the STCs. However, the SDM with the equations in the appendix does not exhibit a good prediction performance under other operating conditions.

To further validate the accuracy of the CS algorithm, the extracted parameters are compared to the ones obtained using GA in Figure 5. In general, the CS algorithm gives the better performance than GA for all cases. The Maximum Power Point (MPP), usually locating around $74 \%$ of the open circuit voltage, is an important technical data in PV modeling. However, a negative point of the GA-based ISDM is that the errors in the high voltage range are relatively high.
The maximum absolute error of the GA-based ISDM is up to about $0.8 \mathrm{~A}$, while the absolute error of the CS is kept below $0.2 \mathrm{~A}$.

\section{Conclusion}

In this work, the Cuckoo Search (CS) algorithm is applied to estimate the parameters of two PV models, namely, Single Diode Model (SDM) and its improved version (ISDM). The feasibility of the proposed method has been validated by estimating the parameters of two commercial PV generators. The simulation and experimental results showed that the CS algorithm is capable of not only extracting all the parameters of the SDM under a certain condition but also successfully 


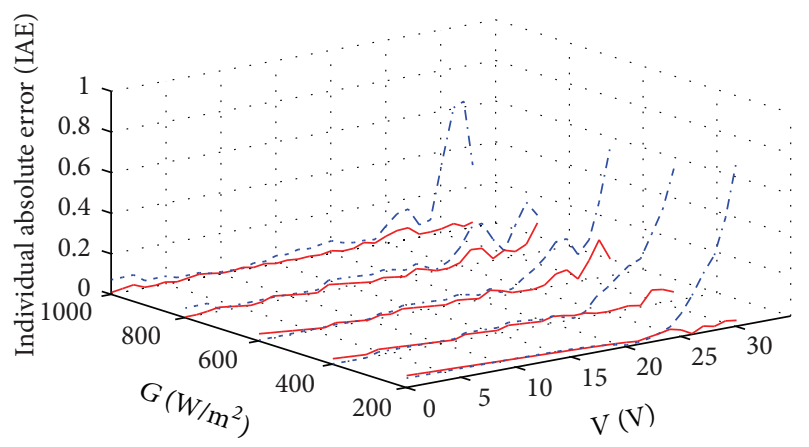

- CS estimation-based ISDM

-. - GA estimation-based ISDM

(a)

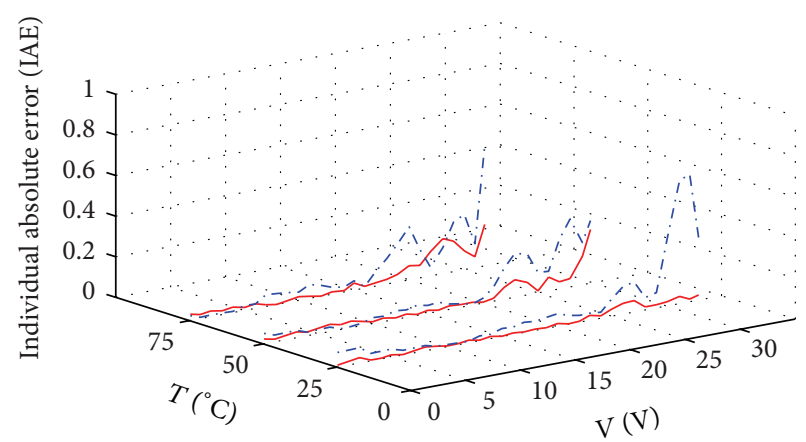

— CS estimation-based ISDM

-. GA estimation-based ISDM

(b)

FIGURE 5: A comparison of the individual absolute errors between CS- and GA- based ISDM: (a) under different irradiance levels; (b) under different temperature levels.

estimating all the parameters of ISDM under different operating conditions. In statistical analysis, CS algorithm recorded the lowest RMSE value compared to other algorithms such as GA, PSO and PS.

\section{Appendix}

\section{PV Physical Model Adopted in PSIM}

By using the parameters extracted at the STCs, the $I-V$ characteristics of a PV generator under nonstandard operating conditions can be calculated via the following equations:

$$
\begin{gathered}
I=I_{\mathrm{pv}}-I_{d}-I_{R}, \\
I_{\mathrm{pv}}=I_{\mathrm{scn}} \cdot \frac{G}{G_{n}}-K_{i} \cdot\left(T-T_{n}\right), \\
I_{d}=I_{o} \cdot\left(e^{q V_{d} / n k T}-1\right), \\
I_{o}=I_{c n} \cdot\left(\frac{G}{G_{n}}\right)^{3} \cdot e^{\left(q E_{g} / n k\right) \cdot\left(1 / T-1 / T_{n}\right)}, \\
I_{R}=\frac{V_{d}}{R_{p}}, \\
V_{d}=\frac{V}{N_{s}}+I \cdot R_{s} .
\end{gathered}
$$

\section{Acknowledgments}

The authors are grateful to Professor Xin-She Yang for the sharing of Cuckoo Search source code online. Without his generosity, this work would not be possible. This research is supported by the National Natural Science Foundation of China under Grant 61070085.

\section{References}

[1] B. Amrouche, A. Guessoum, and M. Belhamel, "A simple behavioural model for solar module electric characteristics based on the first order system step response for MPPT study and comparison," Applied Energy, vol. 91, no. 1, pp. 395-404, 2012.

[2] A. Orioli and A. di Gangi, "A procedure to calculate the fiveparameter model of crystalline silicon photovoltaic modules on the basis of the tabular performance data," Applied Energy, vol. 102 , pp. 1160-1177, 2013

[3] V. Lo Brano, A. Orioli, G. Ciulla, and A. di Gangi, "An improved five-parameter model for photovoltaic modules," Solar Energy Materials and Solar Cells, vol. 94, no. 8, pp. 1358-1370, 2010.

[4] M. G. Villalva, J. R. Gazoli, and E. R. Filho, "Comprehensive approach to modeling and simulation of photovoltaic arrays," IEEE Transactions on Power Electronics, vol. 24, no. 5, pp. 11981208, 2009.

[5] W. De Soto, S. A. Klein, and W. A. Beckman, "Improvement and validation of a model for photovoltaic array performance," Solar Energy, vol. 80, no. 1, pp. 78-88, 2006.

[6] K. Ishaque, Z. Salam, and H. Taheri, "Simple, fast and accurate two-diode model for photovoltaic modules," Solar Energy Materials and Solar Cells, vol. 95, no. 2, pp. 586-594, 2011.

[7] V. Lo Brano, A. Orioli, and G. Ciulla, "On the experimental validation of an improved five-parameter model for silicon photovoltaic modules," Solar Energy Materials and Solar Cells, vol. 105, pp. 27-39, 2012.

[8] A. N. Celik and N. Acikgoz, "Modelling and experimental verification of the operating current of mono-crystalline photovoltaic modules using four- and five-parameter models," Applied Energy, vol. 84, no. 1, pp. 1-15, 2007.

[9] M. A. de Blas, J. L. Torres, E. Prieto, and A. García, "Selecting a suitable model for characterizing photovoltaic devices," Renewable Energy, vol. 25, no. 3, pp. 371-380, 2002.

[10] J. P. Charles, G. Bordure, A. Khoury, and P. Mialhe, "Consistency of the double exponential model with physical mechanisms of conduction for a solar cell under illumination," Journal of Physics D, vol. 18, no. 11, pp. 2261-2268, 1985.

[11] D. S. H. Chan and J. C. H. Phang, "Analytical methods for the extraction of solar-cell single- and double-diode model 
parameters from I-V characteristics," IEEE Transactions on Electron Devices, vol. 34, no. 2, pp. 286-293, 1987.

[12] J. C. H. Phang, D. S. H. Chan, and J. R. Phillips, "Accurate analytical method for the extraction of solar cell model parameters," Electronics Letters, vol. 20, no. 10, pp. 406-408, 1984.

[13] A. J. Joseph, B. Hadj, and A. L. Ali, "Solar cell parameter extraction using genetic algorithms," Measurement Science and Technology, vol. 12, no. 11, pp. 1922-1925, 2001.

[14] W. Huang, C. Jiang, L. Xue, and D. Song, "Extracting solar cell model parameters based on chaos particle swarm algorithm," in Proceedings of the International Conference on Electric Information and Control Engineering (ICEICE '11), pp. 398-402, April 2011.

[15] I. Fister, I. Fister Jr, X. S. Yang, and J. Brest, "A comprehensive review of firefly algorithms," Swarm and Evolutionary Computation, 2013.

[16] M. F. AlHajri, K. M. El-Naggar, M. R. AlRashidi, and A. K. Al-Othman, "Optimal extraction of solar cell parameters using pattern search," Renewable Energy, vol. 44, pp. 238-245, 2012.

[17] A. Askarzadeh and A. Rezazadeh, "Parameter identification for solar cell models using harmony search-based algorithms," Solar Energy, vol. 86, pp. 3241-3249, 2012.

[18] X. Yang and S. Deb, "Engineering optimisation by cuckoo search," International Journal of Mathematical Modelling and Numerical Optimisation, vol. 1, no. 4, pp. 330-343, 2010.

[19] X. Yang and S. Deb, "Cuckoo search via Lévy flights," in Proceedings of the World Congress on Nature and Biologically Inspired Computing (NABIC '09), pp. 210-214, December 2009.

[20] R. A. Messenger and J. Ventre, Photovoltaic Systems Engineering, CRC Press, New York, NY, USA, 2nd edition, 2004.

[21] PSIM User Manual, Powersim, Woburn, Mass, USA, 2001.

[22] R. N. Mantegna, "Fast, accurate algorithm for numerical simulation of Lévy stable stochastic processes," Physical Review E, vol. 49, no. 5, pp. 4677-4683, 1994.

[23] A. H. Gandomi, X. Yang, and A. H. Alavi, "Cuckoo search algorithm: a metaheuristic approach to solve structural optimization problems," Engineering with Computers, vol. 29, pp. 1735, 2013.

[24] B. Birge, Particle Swarm Optimization Toolbox, http://www .mathworks.com/matlabcentral/fileexchange/7506-particleswarm-optimization-toolbox.

[25] P. Civicioglu and E. Besdok, "A conceptual comparison of the Cuckoo-search, particle swarm optimization, differential evolution and artificial bee colony algorithms," Artificial Intelligence Review, vol. 39, pp. 315-346, 2013.

[26] T. Easwarakhanthan, J. Bottin, I. Bouhouch, and C. Boutrit, "Nonlinear minimization algorithm for determining the solar cell parameters with microcomputers," International Journal of Solar Energy, vol. 4, pp. 1-12, 1986.

[27] M. Bashahu and P. Nkundabakura, "Review and tests of methods for the determination of the solar cell junction ideality factors," Solar Energy, vol. 81, no. 7, pp. 856-863, 2007.

[28] S. J. Jun and L. Kay-Soon, "Photovoltaic model identification using particle swarm optimization with inverse barrier constraint," IEEE Transactions on Power Electronics, vol. 27, pp. 3975-3983, 2012.

[29] K. Ishaque, Z. Salam, S. Mekhilef, and A. Shamsudin, "Parameter extraction of solar photovoltaic modules using penaltybased differential evolution," Applied Energy, vol. 99, pp. 297308, 2012.
[30] X. S. Yang, Cuckoo Search Algorithm (Source Code), http:// www.mathworks.com/matlabcentral/fileexchange/29809cuckoo-search-cs-algorithm.

[31] Optimization Toolbox, The MathWorks Inc., http://www .mathworks.com/products/optimization/index.html.

[32] R. L. Burden and J. D. Faires, Numerical Analysis, Cengage Learning, Singapore, 2010. 


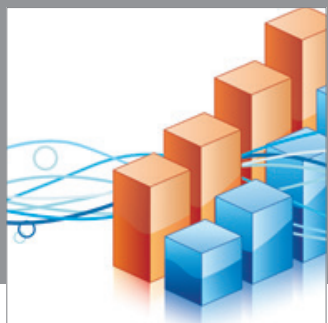

Advances in

Operations Research

mansans

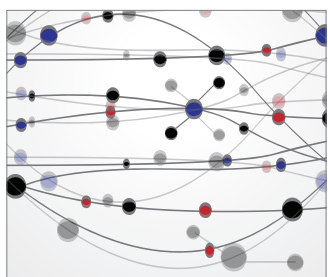

The Scientific World Journal
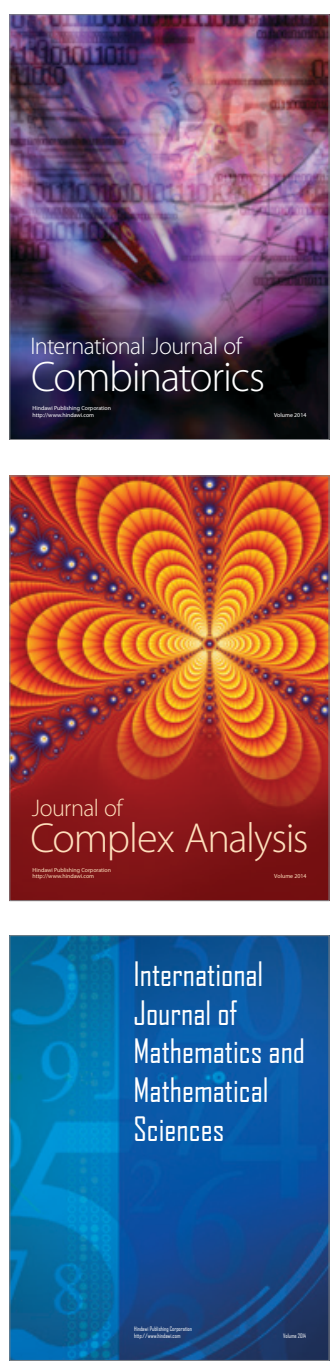
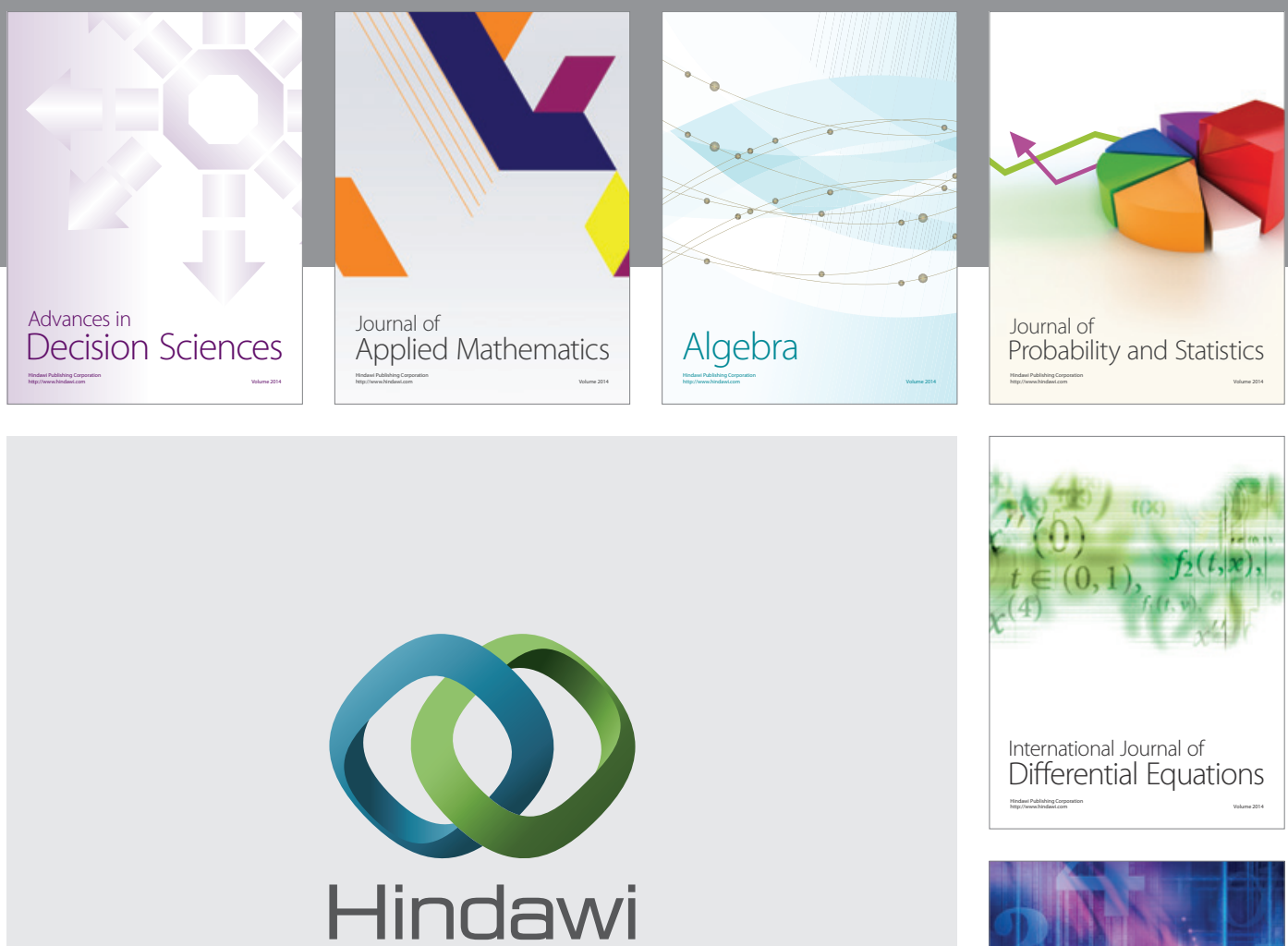

Submit your manuscripts at http://www.hindawi.com
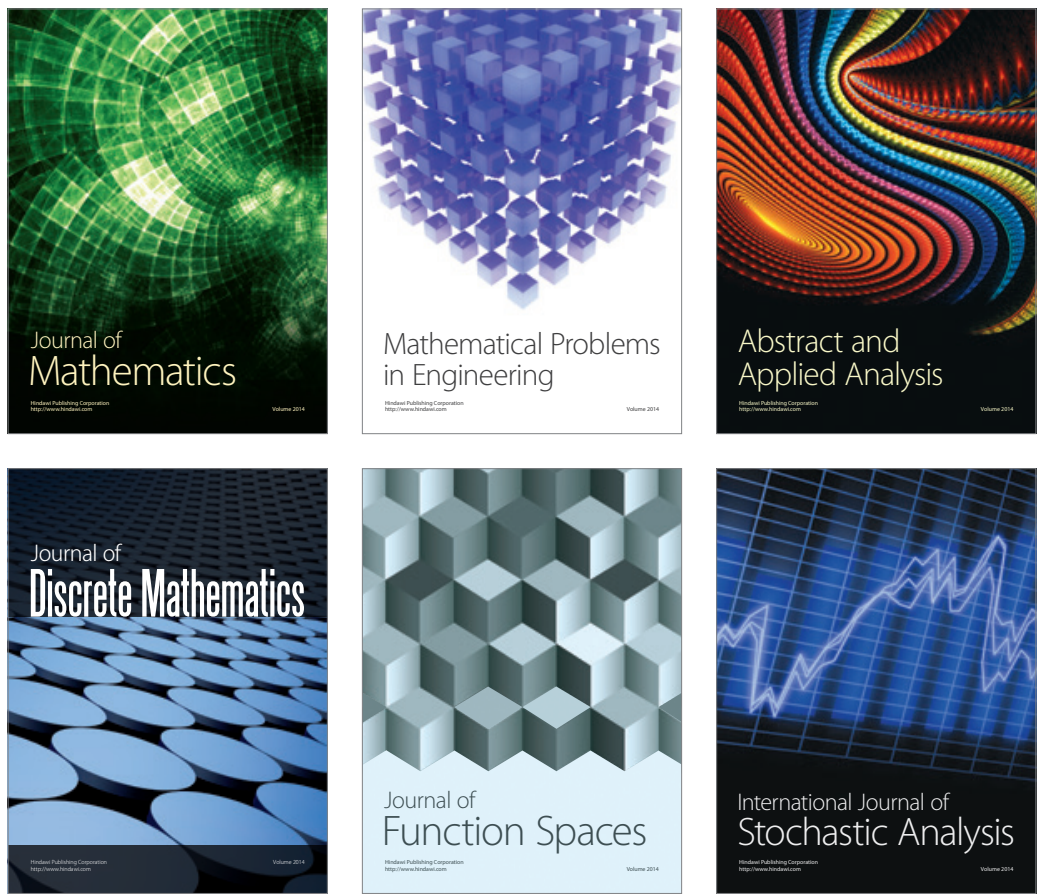

Journal of

Function Spaces

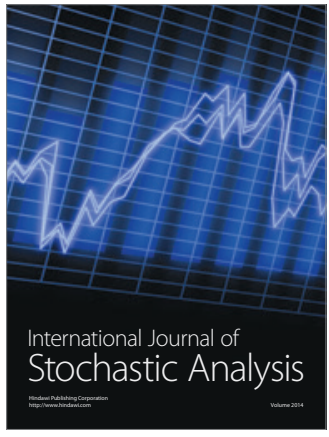

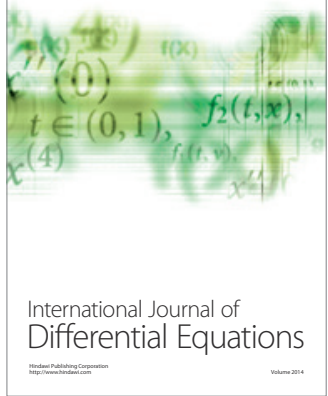
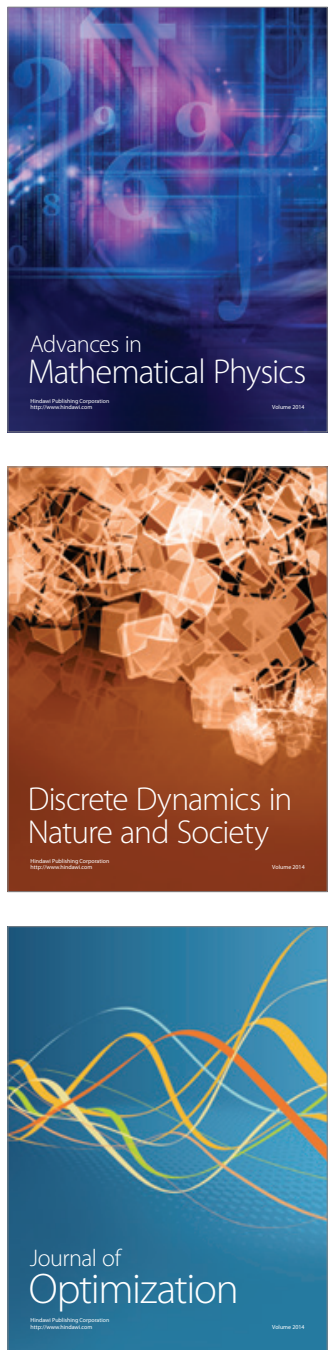